\title{
Determinação simultânea de amarelo tartrazina e amarelo crepúsculo em alimentos via espectrofotometria UV-VIS e métodos de calibração multivariada
}

\author{
Simultaneous determination of tartrazine and sunset yellow in food \\ by spectrophotometry UV-VIS and multivariate calibration methodology
}

\author{
Mara Eliza dos SANTOS ${ }^{1}$, Ivo Mottin DEMIATE ${ }^{2}$, Noemi NAGATA ${ }^{3 *}$
}

\begin{abstract}
Resumo
Os corantes sintéticos são usualmente adicionados a alimentos industrializados para conferir e restaurar a cor obtendo-se a qualidade estética desejada. Em função destes aspectos e do potencial toxicológico que alguns corantes podem apresentar, o controle de qualidade destes compostos é de fundamental importância. Neste trabalho, foi estudado o potencial das metodologias matemáticas como o princípio da aditividade, espectrofotometria derivativa e técnica multivariada (Regressão por Mínimos Quadrados Parciais - PLSR) na determinação simultânea de dois corantes alimentícios: Amarelo Crepúsculo (AC) e Amarelo Tartrazina (AT), extraídos com lã natural. Estas metodologias foram avaliadas e comparadas em função das suas capacidades de previsão, sendo que o modelo PLSR otimizado (faixa espectral de 305 a $645 \mathrm{~nm}$, empregando-se $1^{\text {a }}$ derivada como transformação dos dados e 2 componentes principais) apresentou o menor valor de Raiz Quadrada da Soma dos Erros de Previsão (RMSEP) (AT = 0,191 e AC =0,102). A faixa de concentração estudada foi de 1,0 a 16,0 mg. $\mathrm{L}^{-1}$ para AC e de 2,0 a 22,0 mg. $\mathrm{L}^{-1}$ para AT. O conjunto de validação externa apresentou erros relativos médios de 1,97\% para AC e 1,39\% para AT. A aplicação desta metodologia em amostras reais mostrou que em todas as amostras analisadas as concentrações destes corantes estavam de acordo com os limites estabelecidos pela legislação brasileira.
\end{abstract}

Palavras-chave: PLS; primeira derivada; princípio da aditividade; corantes sintéticos.

\begin{abstract}
Synthetic food dyes are usually added to processed foods to give and restore their desired esthetic quality. This aspect and the toxicological evidence justify quality control and the development of methodologies to quantify these food additives. In this work, the mathematical methodologies such as the additivity principle, derivative spectrophotometry and multivariate technique (Partial Least Square Regression PLSR) were studied in the simultaneous determination of two food colorants: Tartrazine (TY) and Sunset Yellow (SY), extracted from natural wool. These methodologies were evaluated and compared according to their prevision capacities. The best PLSR model (spectral range of 305 to $645 \mathrm{~nm}$ with data transformation by first derivative and two principal components) presented the lower Root Mean Square Error Prediction value, RMSEP $\left(\mathrm{TY}=0.191\right.$ and SY $=0.102$ ). The concentration range was from 1.0 to $16.0 \mathrm{mg} \mathrm{L}^{-1}$ for SY and 2.0 to $22.0 \mathrm{mg} \mathrm{L}^{-1}$ for TY. The validation set was used and showed an average error of $1.97 \%$ for SY and $1.39 \%$ for TY. The application of this methodology in real samples showed concentration values in agreement with the limits established by the Brazilian law and regulations.

Keywords: PLS; first derivative; vierordt's method; synthetic colorants.
\end{abstract}

\section{Introdução}

Os corantes (naturais ou sintéticos) são amplamente utilizados pela indústria de alimentos, visando principalmente conferir, restaurar ou intensificar a cor dos alimentos (BRASIL, 1978), de tal modo que, após o processamento, tenham uma aparência adequada, garantindo a padronização e possibilitando aumentar o número e a variedade de produtos industrializados. Esta adição é justificável, já que a cor é uma das primeiras qualidades sensoriais pelas quais os alimentos são julgados pelos consumidores, sendo associada com a qualidade, $o$ sabor (BERZAS NEVADO; GUIBERTEAU CABANILLAS; CONTENTO SALCEDO, 1995) e até mesmo com o estado de conservação do alimento.
O estudo destes aditivos, tão importantes para a indústria de alimentos, não está somente associado às características benéficas que eles propiciam ao processamento e à venda de produtos alimentícios, mas também pelo seu potencial tóxico à saúde humana. Consequências desta toxicidade podem resultar em alergias e também riscos de câncer (GENNARO; ABRIGO; CIPOLLA, 1994). Em função disto, muitos trabalhos têm sido desenvolvidos para a avaliação da toxicidade destes aditivos (ANTUNES; ARAÚJO, 2000; ELHKIM et al., 2007; LAU et al., 2006), desenvolvimento de metodologias de quantificação (KISELEVA; PIMENOVA; ELLER, 2003; ALGHAMDI, 2005; VIDOTTI et al., 2005), confirmação da presença ou ausência

${ }^{1}$ Mestrado em Ciência e Tecnologia de Alimentos, Universidade Estadual de Ponta Grossa - UEPG

2 Departamento de Engenharia de Alimentos, Universidade Estadual de Ponta Grossa - UEPG

3 Departamento de Química, Centro Politécnico, Universidade Federal do Paraná - UFPR, CP 19081, CEP 81531-990, Curitiba - PR, Brasil, E-mail: nnagata@quimica.ufpr.br ${ }^{*}$ A quem a correspondência deve ser enviada 
em produtos industrializados (PENMAN et al., 2006; CORNET et al., 2006), bem como a estabilidade e produtos de degradação destes corantes durante o processamento e a estocagem dos produtos (GOSETTI et al., 2004; PRABHAKARA RAO; SATYANARAYANA; RAO, 2002; APRUZZESE; BALKE; DIOSADY, 2000).

O controle de qualidade dos corantes tem sido usualmente realizado por técnicas de cromatografia (PRADO; GODOY, 2007; MINIOTI; SAKELLARIOU; THOMAIDIS, 2007; VIDOTTI; COSTA; OLIVEIRA, 2006), eletroforese (PRADO et al., 2006; RYVOLOVA et al., 2007), voltametria (CHANLON et al., 2005; SILVA et al., 2007; DESIMONI; BRUNETTI; COSIO, 2006), ou espectrofotometria (VIDOTTI; ROLLEMBERG, 2006; GÜLER, 2005; ALTINÖZ; TOPTAN, 2002). Nas técnicas instrumentais de separação, características como alto custo instrumental e operacional, incluindo geralmente a necessidade de preparação de amostra, dificultam a obtenção de uma análise rápida. No caso dos métodos espectrofotométricos, embora mais acessíveis, apresentam limitada seletividade decorrente das interferências espectrais que resultam em faixas de absorção amplamente sobrepostas (PERALTA-ZAMORA et al., 1998). No entanto, essa questão de seletividade e sobreposição espectral parcial, pode ser contornada utilizando-se métodos de calibração multivariada para obtenção de um modelo matemático que descreva a relação entre as respostas (absorbâncias) e as concentrações dos componentes das amostras (corantes), minimizando custo e tempo da análise.

A base da calibração multivariada é estabelecer uma relação entre duas matrizes ou blocos de dados químicos, quando houver uma dependência entre as propriedades que descreve cada uma delas. A calibração multivariada consiste basicamente de duas fases: a calibração e a previsão. Quando o estudo é espectroscópico, na fase de calibração, "n" espectros para um conjunto de amostras com composição conhecida são obtidos em "p" valores de comprimento de onda diferentes, formando uma matriz X, com "n" linhas e "p" colunas. Também uma matriz Y pode ser formada, contendo " $n$ " linhas, com os valores de concentração correspondendo às diferentes amostras, "q" colunas, indicando o número de diferentes analitos presentes nas amostras. O próximo passo é desenvolver um modelo matemático apropriado (determinando o vetor dos coeficientes de regressão - b), que melhor possa reproduzir Ycal, a partir dos dados da matriz Xcal (Equação 1). Esse modelo é utilizado na fase de previsão (com um conjunto teste), para estimar as concentrações (Yprev) dos constituintes de novas amostras, a partir de seus espectros (Xteste) (Equação 2). Como estas metodologias trabalham com matrizes de dados, o processo de isolar o fator Y da Equação 1 para obtenção da Equação 2 implica na utilização da matriz transposta de X, ou seja, (Xteste)t.

$\mathrm{Xcal}=\mathrm{b} *$ Ycal

Yteste $=(\text { Xteste })^{\mathrm{t} * \mathrm{~b}}$

A aplicação destas metodologias multivariadas em corantes de interesse para a indústria alimentícia tem se mostrado propícia para diversas técnicas instrumentais (DINC; AKTAS; USTUNDAG, 2005; KARAOGLAN et al., 2007;
BERZAS NEVADO et al., 1999; LOPEZ-DE-ALBA; LOPEZMARTINEZ; DE-LEON-RODRIGUEZ, 2002), evidenciado sua potencialidade para aplicação no controle de qualidade de diversas amostras comerciais.

Em função das características positivas que os métodos multivariados têm apresentado, este trabalho tem a finalidade de comparar os resultados obtidos para a determinação simultânea de Amarelo Tartrazina e Amarelo Crepúsculo através da metodologia de Regressão de Mínimos Quadrados Parciais (PLSR) e duas metodologias matemáticas convencionais (primeira derivada dos dados espectrais e princípio da aditividade espectrofotométrica). O modelo de melhor desempenho para esta quantificação será aplicado à determinação dos corantes em amostras alimentícias comerciais.

\section{Material e métodos}

\subsection{Material}

Os padrões de corantes sintéticos AT e AC foram gentilmente cedidos pela Kerry do Brasil Ltda. e Alimentas Engenharia \& Tecnologia Ltda. Todos os reagentes utilizados no preparo de soluções e nos procedimentos de extração e eluição dos corantes em produtos alimentícios foram de grau analítico P.A, sendo a água destilada proveniente de um destilador de vidro. A lã natural empregada na extração dos corantes das matrizes alimentícias foi previamente tratada com $\mathrm{NH}_{4} \mathrm{OH}$ até que a solução de lavagem não apresentasse sinais de coloração.

\subsection{Métodos}

\section{Amostras reais}

Para o preparo das amostras, foi utilizado o método de extração em lã, no qual alíquotas das amostras foram transferidas para um béquer de $100 \mathrm{~mL}$ e cerca de $50 \mathrm{~mL}$ de água destilada foram adicionados juntamente com cinco gotas de ácido clorídrico concentrado e pedaços de lã. Essa solução foi agitada por alguns minutos, até que se obtivesse uma solução clara e desprovida de cor. Em seguida, a lã colorida foi lavada abundantemente com água destilada e transferida para um béquer de $100 \mathrm{~mL}$, juntamente com $20 \mathrm{~mL}$ de solução a tampão $\mathrm{NH}_{4} \mathrm{OH} / \mathrm{NH}_{4} \mathrm{Cl}$ pH 10. À medida que a solução tampão eluia o corante da lã, esta solução era transferida para balões volumétricos. Esse procedimento foi repetido até que a lã não desprendesse mais cor. Por fim aferiu-se o balão com solução tampão para posterior leitura em espectrofotômetro UV-VIS SCINCO S1150.

\section{Princípio da aditividade espectrofotométrica}

As absortividades molares de cada um dos corantes (AT e AC) foram obtidas a partir das curvas analíticas das soluções padrões do corante AT (na faixa de concentração 30,0 a $120 \mathrm{mg} . \mathrm{L}^{-1}$ ) e AC (na faixa de concentração 10,0 a 50,0 mg. $\mathrm{L}^{-1}$ ) em solução tampão $\mathrm{NH}_{4} \mathrm{OH} / \mathrm{NH}_{4} \mathrm{Cl}(\mathrm{pH} 10)$. 


\section{Espectrofotometria derivativa}

Para determinação do ponto de inflexão (derivada da absorbância igual a zero, independente da concentração do analito) de cada um dos corantes, espectros das soluções padrões do corante AT (na faixa de concentração 30,0 a 120 mg. $\mathrm{L}^{-1}$ ) e AC (na faixa de concentração 10,0 a 50,0 mg. $\mathrm{L}^{-1}$ ) em tampão de $\mathrm{NH}_{4} \mathrm{OH} / \mathrm{NH}_{4} \mathrm{Cl}(\mathrm{pH}$ 10) foram obtidos na faixa de 200 a $800 \mathrm{~nm}$, sendo posteriormente derivados e alisados empregando-se o programa Microcal Origin ${ }^{\mathrm{TM}}$ (Versão 6.1). Posteriormente, curvas analíticas (dA versus Concentração) foram obtidas para a faixa de concentração de interesse: AT de 2,0 a 22,0 mg. $\mathrm{L}^{-1} \mathrm{e}$ AC de 1,0 a 16,0 mg. $\mathrm{L}^{-1}$.

\section{Calibração multivariada}

Foram preparadas 36 misturas sintéticas em meio tamponado $\mathrm{NH}_{4} \mathrm{OH} / \mathrm{NH}_{4} \mathrm{Cl}$ ( $\left.\mathrm{pH} 10\right)$, cada uma delas composta por diferentes proporções dos padrões AT e AC. Para todas as misturas, quantidades conhecidas das soluções estoque de $200 \mathrm{mg} . \mathrm{L}^{-1}$ de AT e $100 \mathrm{mg} . \mathrm{L}^{-1}$ de AC foram utilizadas. Então, alíquotas destas soluções foram transferidas para um balão volumétrico de $10,0 \mathrm{~mL}$ e completado até o volume final com tampão $\mathrm{pH}$ 10. O planejamento experimental utilizado para compor as 30 misturas sintéticas responsáveis pelo desenvolvimento dos modelos de calibração e as seis misturas que compõem o conjunto de validação pode ser visualizado na Figura 1. A faixa de concentração estudada foi de 2,0 a 22,0 mg.L. $\mathrm{L}^{-1}$ para o AT e de 1,0 a 16,0 mg. $\mathrm{L}^{-1}$ para o AC.

Posteriormente, os espectros UV-VIS (Espectrofotômetro SCINCO, modelo S1150) destas 36 misturas sintéticas foram coletados, sendo que apenas 30 destes padrões sintéticos foram utilizados para desenvolver os modelos de calibração multivariada (PLSR). Todos os dados espectrais foram transportados em formato ASCII pelo programa Microcal Origin $^{\text {TM }}$ (Versão 6.1) e a análise multivariada dos dados foi realizada empregando-se o programa Matlab for Windows (versão 4.0) dotado do pacote PLS Toolbox (versão 1.5).

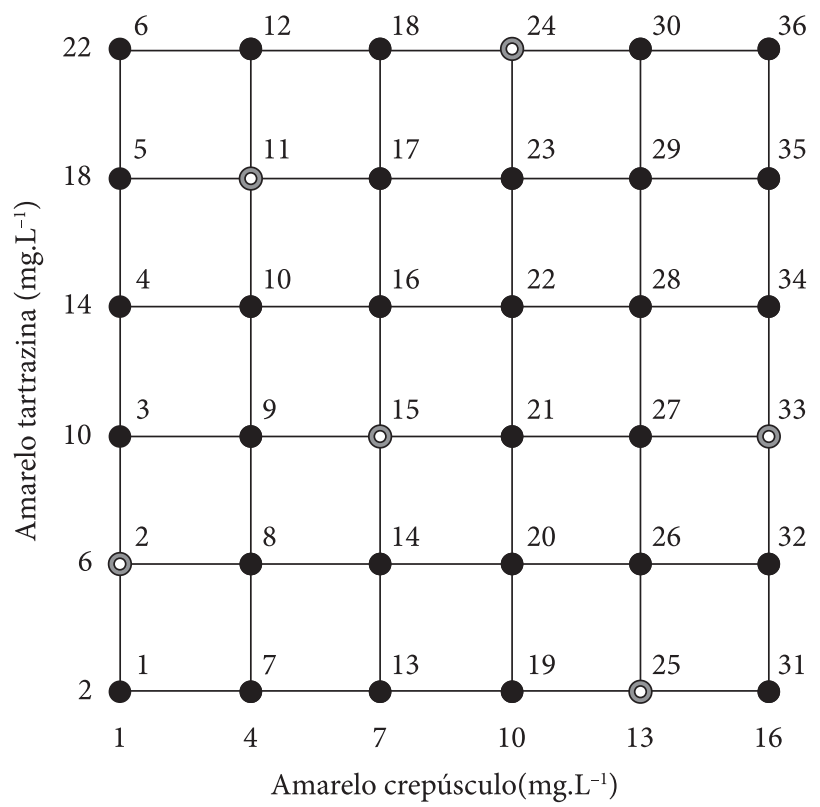

Figura 1. Composição das misturas sintéticas do conjunto de calibração (•) e previsão (@).

\section{Validação dos modelos}

Esta etapa de validação foi realizada para as três metodologias matemáticas de quantificação propostas neste trabalho: princípio da aditividade, primeira derivada e calibração multivariada. Após elaboração de vários modelos de calibração, a capacidade de previsão de cada um deles foi avaliada frente a seis padrões sintéticos, de concentrações diferentes daquelas adotadas na fase de calibração (Figura 1, amostras destacadas em cinza). Com base nestes resultados, foi escolhido o modelo de melhor desempenho, que foi utilizado na determinação das espécies de interesse em produtos comerciais.

\section{Resultados e discussão}

\subsection{Princípio da aditividade espectrofotométrica}

Para a utilização do princípio da aditividade na análise espectrofotométrica simultânea dos corantes, curvas analíticas independentes foram construídas nos dois comprimentos de onda no qual se encontram as absorções máximas dos corantes AT e AC (402 e $460 \mathrm{~nm}$, respectivamente) com o objetivo de determinar suas absortividades $(\varepsilon)$. Estes dados são mostrados na Tabela 1.

Considerando-se que as únicas espécies responsáveis pela absorção na região de 402 e $460 \mathrm{~nm}$ sejam os corantes de interesse, a aplicação da aditividade da lei de Beer $(A=\varepsilon b c)$ pode ser obtida pela junção dos dados (Tabela 1), a qual permite obter uma equação que engloba a influência do corante AT e AC no comprimento de onda $402 \mathrm{~nm}$ (Equação 3) e outra em 460 nm (Equação 4), respectivamente.

$\mathrm{A}_{402}=0,823 \mathrm{c}_{\mathrm{AT}}+1,449 \mathrm{c}_{\mathrm{AC}}$

$\mathrm{A}_{460}=0,424 \mathrm{c}_{\mathrm{AT}}+2,819 \mathrm{c}_{\mathrm{AC}}$

A partir do espectro da mistura destes corantes é possível determinar $\mathrm{A}_{402}$ e $\mathrm{A}_{460}$ e, assim, a resolução das equações simultâneas descritas anteriormente possibilita determinar a concentração de AT e AC na amostra analisada.

Para avaliar a capacidade de previsão do método espectrofotométrico associado ao princípio da aditividade, um conjunto de validação contendo a mistura dos corantes foi analisado (Figura 1, amostras destacadas em cinza). Os resultados obtidos estão apresentados na Tabela 2, juntamente com os dados de RMSEP (Raiz Quadrada da Soma dos Erros de Previsão) para cada corante. O RMSEP é uma forma de avaliar o erro absoluto e, portanto, permite uma visualização e conclusão rápida do aumento ou diminuição na exatidão de cada uma das metodologias matemáticas empregadas.

Tabela 1. Curvas de calibração obtidas para os corantes AT e AC em 402 e $460 \mathrm{~nm}$.

\begin{tabular}{ccc}
\hline Corantes & Equações & $\mathrm{R}^{2}$ \\
\hline Tartrazina $(402 \mathrm{~nm})$ & $\mathrm{A}_{402}=0,823[\mathrm{AT}]-0,051$ & 0,9984 \\
Tartrazina $(460 \mathrm{~nm})$ & $\mathrm{A}_{460}=0,424[\mathrm{AT}]+0,027$ & 0,9976 \\
Crepúsculo $(402 \mathrm{~nm})$ & $\mathrm{A}_{402}=1,449[\mathrm{AC}]+0,035$ & 0,9988 \\
Crepúsculo $(460 \mathrm{~nm})$ & $\mathrm{A}_{460}=2,819[\mathrm{AC}]+0,003$ & 0,9991 \\
\hline
\end{tabular}


A aplicação do princípio da aditividade para determinação da mistura dos corantes de interesse evidencia a tendência de um erro sistemático negativo, que subestima a determinação de ambos corantes. Esta tendência deve ser decorrente da sobreposição considerável entre os máximos de absorção de ambos analitos, já que uma maior exatidão em uma análise deste tipo é conseguida quando existe uma diferença elevada entre as absortividades molares dos compostos em um mesmo comprimento de onda.

\subsection{Espectrofotometria derivativa}

Inicialmente foram determinados os pontos de inflexão para os corantes AT e AC, separadamente. A Figura 2 mostra os espectros derivados de AT (em preto) em diversas concentrações, em que é possível evidenciar apenas um ponto de inflexão, ou seja, um comprimento de onda $(406,45 \mathrm{~nm})$ no qual a concentração de AT não influencia no valor da derivada da absorbância, resultando sempre em valor zero. Isso possibilita a determinação de AC neste comprimento de onda, "sem" interferência da concentração de AT.

No caso do AC (em cinza), dois pontos de inflexão são passíveis de serem observados, ou seja, dois comprimentos de onda (364,76 e 466,92 nm) em que a concentração de AC não influencia na medida da derivada de absorção do AT.

Para aplicação desta metodologia, foram obtidas curvas analíticas a partir de soluções padrão dos corantes de interesse contendo entre quatro e seis pontos de diferentes concentrações. Os valores da derivada da absorbância do espectro foram medidos em 406,45 nm para o corante AC e 364,76 e 466,92 nm para o corante AT. O fato de o corante AT apresentar a possibilidade de leitura em dois comprimentos de onda distintos

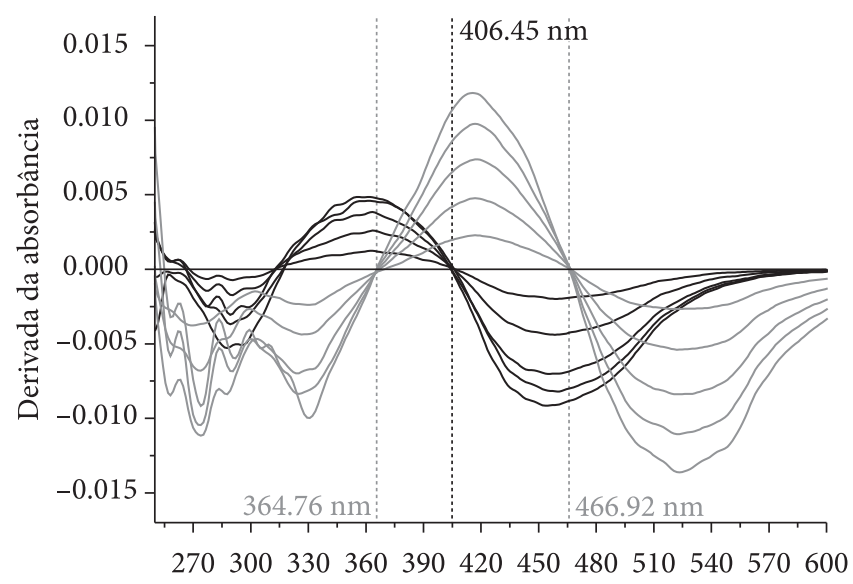

$$
\begin{aligned}
\text { Comprimento de onda }(\mathrm{nm}) \\
-[\mathrm{AC}]=10 \mathrm{mg} \cdot \mathrm{L}^{-1}-[\mathrm{AT}]=30 \mathrm{mg} \cdot \mathrm{L}^{-1} \\
-[\mathrm{AC}]=20 \mathrm{mg} \cdot \mathrm{L}^{-1}-[\mathrm{AT}]=60 \mathrm{mg} \cdot \mathrm{L}^{-1} \\
-[\mathrm{AC}]=30 \mathrm{mg} \cdot \mathrm{L}^{-1}-[\mathrm{AT}]=90 \mathrm{mg} \cdot \mathrm{L}^{-1} \\
-[\mathrm{AC}]=40 \mathrm{mg} \cdot \mathrm{L}^{-1}-[\mathrm{AT}]=105 \mathrm{mg} \cdot \mathrm{L}^{-1} \\
-[\mathrm{AC}]=50 \mathrm{mg} \cdot \mathrm{L}^{-1}-[\mathrm{AT}]=120 \mathrm{mg} \cdot \mathrm{L}^{-1}
\end{aligned}
$$

Figura 2. Espectros derivados e alisados dos corantes AT e AC em diversas concentrações. permitiu a construção de duas curvas de calibração. A Tabela 3 apresenta os parâmetros (curvas de calibração, coeficientes de correlação e os respectivos comprimentos de onda) que possibilitam a determinação simultânea dos corantes AT e AC via metodologia da $1^{\text {a }}$ derivada.

No método da primeira derivada, o mesmo conjunto de validação contendo as misturas binárias dos padrões sintéticos de AT e AC foi utilizado para verificar o potencial de previsão desta metodologia. Aplicando-se o valor da derivada da absorbância (no comprimento de onda determinado) na sua respectiva equação (Tabela 3), é possível obter a concentração dos corantes de interesse. A Tabela 4 apresenta os resultados desta previsão e de RMSEP para os corantes: amarelo tartrazina e amarelo crepúsculo.

\subsection{Modelo de calibração multivariada (PLSR)}

A metodologia de calibração multivariada foi conduzida essencialmente via processos de Regressão de Mínimos Quadrados Parciais (PLSR). O modelo foi desenvolvido a partir do planejamento experimental contendo 36 misturas binárias como mostrado na Figura 1. Deste conjunto, 30 padrões foram utilizados para a etapa de calibração (conjunto de calibração) e seis padrões para a fase de validação (conjunto de validação). $\mathrm{O}$ modelo de calibração otimizado foi processado na faixa espectral do visível, compreendida entre 305 a 625 nm (localização do sinal analítico do principal grupo cromóforo), empregando-se $1^{\text {a }}$ derivada aos dados espectrais, pré-processamento com dados centrados na média e procedimento de validação cruzada.

No procedimento de validação cruzada, a calibração pode ser repetida $n$ vezes ( $n=$ número de amostras), sendo que em cada oportunidade uma das amostras do conjunto de calibração é retirada e utilizada como amostra de previsão. Uma vez que todas as amostras tenham sido tratadas como objeto de previsão, é possível estimar a Soma dos Quadrados dos Erros de Previsão

Tabela 2. Resultados de previsão e RMSEP para o princípio da

\begin{tabular}{|c|c|c|c|}
\hline \multicolumn{2}{|c|}{ Tartrazina $\left(\mathrm{mg} . \mathrm{L}^{-1}\right)$} & \multicolumn{2}{|c|}{ Crepúsculo (mg.L-1 $)$} \\
\hline Valor real & Valor previsto & Valor real & Valor previsto \\
\hline 6,0 & 6,00 & 1,0 & 0,92 \\
\hline 18,0 & 15,7 & 4,0 & 3,87 \\
\hline 10,0 & 9,78 & 7,0 & 6,52 \\
\hline 22,0 & 19,5 & 10,0 & 9,14 \\
\hline 2,0 & 1,74 & 13,0 & 11,9 \\
\hline 10,0 & 8,11 & 16,0 & 14,6 \\
\hline RMSEP* $^{*}$ & 1,97 & RMSEP* & 0,86 \\
\hline
\end{tabular}
aditividade.

Tabela 3. Parâmetros para determinação de AT e AC via primeira derivada.

\begin{tabular}{ccr}
\hline $\begin{array}{c}\text { Comprimento de } \\
\text { onda }(\mathrm{nm})\end{array}$ & \multicolumn{1}{c}{$\begin{array}{c}\text { Curva de } \\
\text { calibração }\end{array}$} & $\mathrm{R}^{2}$ \\
\hline 364,76 & $\mathrm{dA} / \mathrm{d}[\mathrm{AT}]=-8,77 \mathrm{E}-5+4,16 \mathrm{E}-5[\mathrm{AT}]$ & 0,99770 \\
466,92 & $\mathrm{dA} / \mathrm{d}[\mathrm{AT}]=-8,06 \mathrm{E}-5-6,57 \mathrm{E}-5[\mathrm{AT}]$ & $-0,99460$ \\
406,45 & $\mathrm{dA} / \mathrm{d}[\mathrm{AC}]=-4,59 \mathrm{E}-5+20,4 \mathrm{E}-5[\mathrm{AC}]$ & 0,99993 \\
\hline
\end{tabular}


(PRESS) dada pela Equação 5 e escolher a quantidade de componentes principais necessária para a obtenção do menor PRESS.

PRESS $=\sum\left(\mathrm{C}_{\text {Real }}-\mathrm{C}_{\text {Prevista }}\right)^{2}$

A partir deste procedimento foi possível verificar que após a $2^{\text {a }}$ componente principal não existe nenhum ganho significativo em termos de minimização de PRESS. Além disso, estas duas primeiras componentes são responsáveis pela explicação de cerca de 99,80\% da variância dos dados, fator que é concordante com o número de corantes que variam sua concentração no conjunto de calibração.

O gráfico de coeficientes de regressão (Figura 3) indica que a região espectral utilizada para a construção do modelo (305$645 \mathrm{~nm}$ ) apresenta informação analítica relevante para o controle quantitativo em questão. Além disso, nos locais onde aparecem as bandas de absorção dos corantes na região do visível, há um aumento do coeficiente de regressão (destacado com ${ }^{\star}$ ).

A capacidade de previsão deste modelo pode ser visualizada na Figura 4, cujas concentrações previstas são muito próximas às concentrações dos padrões do conjunto de calibração $\left(r^{2} \approx 0,999\right.$

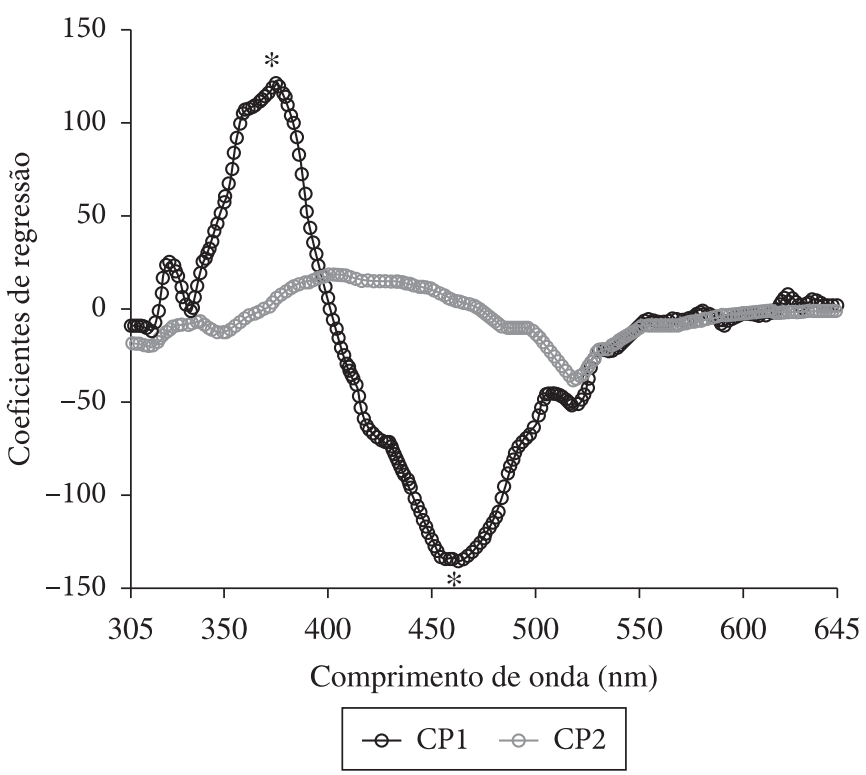

Figura 3. Coeficientes de regressão do modelo PLSR com derivada dos dados. para ambos corantes). Através dos valores de Raiz Quadrada da Soma dos Erros de Calibração (RMSEC) e Raiz Quadrada da Soma dos Erros de Validação Cruzada (RMSECV), é possível observar uma maior exatidão na determinação do corante AC $(\mathrm{RMSEC}=0,1781$ e RMSECV $=0,1999)$ do que para o corante AT (RMSEC = 0,3169 e RMSECV = 0,3596). O modelo engloba uma ampla faixa de concentração respondendo satisfatoriamente mesmo em concentrações mais baixas.

A análise dos resíduos em função de leverage (medida de influência de uma amostra no modelo de regressão) indica que nenhuma amostra do conjunto de calibração apresenta-se fora dos limites comumente aceitos. Todas as amostras apresentam resíduos studentizados aleatórios menores que 2,5 e leverage menor que $3 \mathrm{p} / \mathrm{n}$ (sendo $\mathrm{p}$ corresponde ao número de componentes principais consideradas para a otimização do modelo e $\mathrm{n}$ ao número de padrões de calibração, ou seja, 0,2). Não há, portanto, evidências da existência de qualquer amostra anômala (outlier) no conjunto utilizado para construir o modelo.

Findado o processo de otimização do modelo PLSR, este foi testado aos dados espectrais do conjunto de validação. A Tabela 5 apresenta os valores de erros relativos obtidos na análise de corantes utilizando o modelo PLSR já descrito.

Avaliando-se a capacidade de previsão do modelo PLSR desenvolvido através do teste $\mathrm{F}\left(\mathrm{F}_{29,5,95 \%}=4,50\right)$, foi possível observar que nenhuma diferença estatística na previsão foi encontrada entre o procedimento de calibração (RMSEC) e validação externa (RMSEP) com 95\% de confiança.

Os resultados indicam baixos erros relativos tanto na determinação de AC quanto de AT, sendo que na maioria dos casos os maiores erros relativos estão associados às menores concentrações dos corantes analisados.

Tabela 5. Resultados de Previsão e RMSEP na análise de corantes (mg.L $\mathrm{L}^{-1}$ ) por PLSR.

\begin{tabular}{cccccc}
\hline $\begin{array}{c}\text { Valor real } \\
(\mathrm{AT})\end{array}$ & $\begin{array}{c}\text { Valor } \\
\text { previsto }\end{array}$ & $\begin{array}{c}\text { Erro } \\
\text { relativo }\end{array}$ & $\begin{array}{c}\text { Valor real } \\
(\mathrm{AC})\end{array}$ & $\begin{array}{c}\text { Valor } \\
\text { previsto }\end{array}$ & $\begin{array}{c}\text { Erro } \\
\text { relativo }\end{array}$ \\
\hline 6,0 & 5,75 & $-4,17$ & 1,0 & 1,03 & 3,00 \\
18,0 & 17,9 & $-0,56$ & 4,0 & 4,05 & 1,25 \\
10,0 & 10,4 & 4,00 & 7,0 & 7,05 & 0,71 \\
22,0 & 22,1 & 0,45 & 10,0 & 10,1 & 1,00 \\
2,0 & 2,05 & 2,50 & 13,0 & 13,2 & 1,54 \\
10,0 & 10,1 & 1,00 & 16,0 & 16,1 & 0,63 \\
RMSEP & 0,28 & & RMSEP & 0,14 & \\
\hline
\end{tabular}

Tabela 4. Resultados de Previsão e RMSEP para primeira derivada.

\begin{tabular}{|c|c|c|c|}
\hline $\begin{array}{c}\text { Valores reais AT - AC } \\
\left(\mathrm{mg} \cdot \mathrm{L}^{-1}\right)\end{array}$ & $\begin{array}{c}\text { Valor previsto AT - 364,76 nm } \\
\left(\mathrm{mg} . \mathrm{L}^{-1}\right)\end{array}$ & $\begin{array}{c}\text { Valor previsto AT - 466,92 nm } \\
\left({\left.\mathrm{mg} . \mathrm{L}^{-1}\right)}\right.\end{array}$ & $\begin{array}{c}\text { Valor previsto AC - 406,45 nm } \\
\left(\mathrm{mg} \cdot \mathrm{L}^{-1}\right)\end{array}$ \\
\hline $6,0-1,0$ & 6,56 & 8,98 & 0,93 \\
\hline $18,0-4,0$ & 18,0 & 19,9 & 3,97 \\
\hline $10,0-7,0$ & 11,3 & 11,5 & 7,12 \\
\hline $22,0-10,0$ & 25,1 & 21,7 & 9,96 \\
\hline $2,0-13,0$ & 2,19 & 0,88 & 13,3 \\
\hline $10,0-16,0$ & 12,3 & 7,80 & 16,4 \\
\hline RMSEP & 1,97 & 2,32 & 0,19 \\
\hline
\end{tabular}




\subsection{Comparação entre as metodologias matemáticas estudadas}

As Tabelas 2, 4 e 5 apresentam os resultados de RMSEP para as três metodologias matemáticas estudadas: Método da aditividade, $1^{\text {a }}$ Derivada e Metodologia Multivariada (PLSR). Os resultados obtidos destacam a metodologia multivariada como a técnica de melhor desempenho na determinação quantitativa simultânea de AT e AC.

\subsection{Aplicação do modelo PLSR na determinação de AT e AC em amostras alimentícias}

Tendo em vista os resultados dos modelos construídos e considerando-se o valor de RMSEP como parâmetro para escolha do modelo com a melhor capacidade de previsão, $\mathrm{o}$ modelo otimizado PLSR foi utilizado na determinação dos corantes AT e AC em amostras de alimentos.

Vale a pena ressaltar que como um processo de extração em lã foi adotado para evitar a interferência de outros componentes das amostras de alimentos na determinação espectrofotométrica desejada, o fator de recuperação de cada um dos corantes eluído com solução tampão $\mathrm{NH}_{4} \mathrm{OH} / \mathrm{NH}_{4} \mathrm{Cl}$ foi determinado previamente.

A Tabela 6 apresenta a média (triplicatas) dos teores dos corantes AT e AC em amostras de alimentos, já corrigidos pelo fator de recuperação, para cada um dos três lotes analisados.

Os resultados de todos os controles realizados estão dentro dos limites estabelecidos pela legislação. Para exemplificar a Dose Diária Admitida (DDA) para o corante AT, considerando uma pessoa com massa corporal aproximada de $60 \mathrm{~kg}$, seria necessário que a mesma ingerisse em um dia cerca de $26 \mathrm{~L}$ (52 caixas) de gelatina em pó sabor abacaxi para ultrapassar o limite estabelecido na JECFA. No caso da DDA para o corante AC, seria necessário que a pessoa ingerisse cerca de $69 \mathrm{~L}$ (69 pacotes) de suco em pó de laranja para ultrapassar o limite estabelecido.

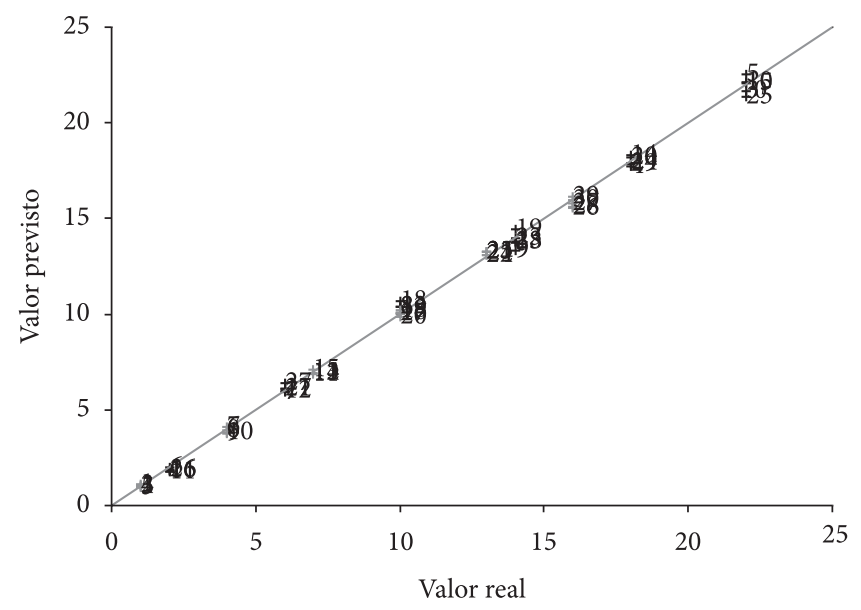

Figura 4. Valores reais versus valores previstos pelo modelo PLSR com derivada dos dados e faixa espectral de 305 a $625 \mathrm{~nm}$.
Tabela 6. Média dos teores de corantes artificiais (mg. $\left.\mathrm{L}^{-1}\right)$ em amostras de alimentos.

\begin{tabular}{cccc}
\hline Amostras / Sabor & Lotes & Tartrazina $^{\star}$ & Crepúsculo $^{* *}$ \\
\hline Gelatina abacaxi & 1 & $17,97 \pm 0,39$ & $1,85 \pm 0,12$ \\
& 2 & $17,54 \pm 1,63$ & $1,69 \pm 0,30$ \\
Suco em pó laranja & 3 & $16,98 \pm 1,52$ & $1,54 \pm 0,10$ \\
& 1 & $12,23 \pm 0,97$ & $2,13 \pm 0,12$ \\
Suco líquido abacaxi & 2 & $10,91 \pm 0,22$ & $2,18 \pm 0,06$ \\
& 3 & $9,80 \pm 2,04$ & $1,95 \pm 0,24$ \\
Isotônico laranja & 1 & $6,14 \pm 0,57$ & $0,33 \pm 0,02$ \\
& 3 & $5,87 \pm 0,96$ & $0,20 \pm 0,10$ \\
& 1 & $5,68 \pm 0,29$ & $0,28 \pm 0,05$ \\
& 2 & $16,07 \pm 0,53$ & $1,71 \pm 0,11$ \\
& 3 & $16,06 \pm 0,19$ & $1,54 \pm 0,08$ \\
\hline
\end{tabular}

* Corrigidos por $90 \%$ de recuperação; ${ }^{* *}$ Corrigidos por $86 \%$ de recuperação.

\section{Conclusões}

Em função do conjunto de resultados conseguido neste trabalho, é possível concluir que, tal como esperado e observado por outros grupos de pesquisa, a análise de corantes empregando-se espectrofotometria UV-VIS acoplada a metodologias matemáticas permite uma adequada determinação de multicomponentes em matrizes alimentares.

No processo de quantificação dos corantes de interesse, duas metodologias matemáticas convencionais (princípio da aditividade espectrofotométrica e $1^{\text {a }}$ derivada) e uma multivariada (PLSR) foram empregadas. Os resultados foram avaliados e comparados utilizando-se um conjunto de validação externa contendo seis misturas sintéticas (concentrações conhecidas), pela determinação da exatidão (RMSEP) de cada uma das metodologias.

No caso das metodologias matemáticas convencionais, a análise de AT apresentou RMSEP de 1,97 para a primeira derivada e o princípio da aditividade, enquanto para a análise de AC obteve-se um RMSEP de 0,19 para a primeira derivada e 0,86 para o princípio da aditividade.

O modelo de melhor capacidade de previsão foi o PLSR, cuja transformação dos dados espectrais (faixa de 305-645 nm) em primeira derivada e pré-processamento com dados centrados na média propiciou RMSEP de 0,28 e 0,14 para AT e AC, respectivamente.

Em função destes resultados, o modelo multivariado PLSR foi utilizado no controle de qualidade dos corantes de interesse em produtos alimentícios e todas as amostras analisadas apresentaram valores de AT e AC em concordância com a legislação brasileira vigente.

\section{Agradecimentos}

Os autores agradecem ao Prof. Dr. Patricio G. PeraltaZamora (DEQUIM-UFPR) a disponibilização da infraestrutura necessária para o desenvolvimento deste trabalho, ao órgão de fomento à pesquisa Capes o auxílio concedido na forma de bolsa de mestrado e à Fundação Araucária - PR/Apoio à Instalação de Recém-Doutores (Chamada 04/01 - Protocolo 1017) o apoio financeiro. 


\section{Referências bibliográficas}

ALGHAMDI, A. H. Determination of Allura Red in some food samples by adsorptive stripping voltammetry. Journal of AOAC International, v. 88, n. 5, p. 1387-1393, 2005.

ALTINÖZ, S.; TOPTAN, S. Determination of Tartrazine and Ponceau4R in Various Food Samples by Vierordt's Method and Ratio Spectra First-Order Derivative UV Spectrophotometry. Journal of Food Composition and Analysis, v. 15, n. 6, p. 667-683, 2002.

ANTUNES, L. M. G.; ARAÚJO, M. C. P. Mutageniciddade e antimutagenicide dos principais corantes para alimentos. Revista de Nutrição, v. 13, n. 2, p. 81-88, 2000.

APRUZZESE, F.; BALKE, S. T.; DIOSADY, L. L. In-line colour and composition monitoring in the extrusion cooking process. Food Research International, v. 33, n. 7, p. 621-628, 2000.

BERZAS NEVADO, J. J. et al. Simultaneous spectrophotometric determination of tartrazine, patent blue $\mathrm{V}$, and indigo carmine in commercial products by partial least squares and principal component regression methods. Talanta, v. 48, n. 4, p. 895-903, 1999.

BERZAS NEVADO, J. J.; GUIBERTEAU CABANILLAS, C.; CONTENTO SALCEDO, A. M. Simultaneous spectrophotometric determination of three food dyes by using the first derivative of ratio spectra. Talanta, v. 42, n. 12, p. 2043-2051, 1995.

BRASIL. Agência Nacional de Vigilância Sanitária - ANVISA. Resolução - CNNPA nº 44, de 1977. Diário Oficial [da] República Federativa do Brasil, Brasília, DF, 24 abr. 1978. Seção 1. Disponível em: <http://www.anvisa.gov.br/legis/resol/44_77.htm>. Acesso em: 14 jul. 2008.

CHANLON, S. et al. Determination of Carmoisine, Allura red and Ponceau $4 \mathrm{R}$ in sweets and soft drinks by Differential Pulse Polarography. Journal of Food Composition and Analysis, v. 18, n. 6, p. 503-515, 2005.

CORNET, V. et al. Development of a fast analytical method for the determination of sudan dyes in chili- and curry-containing foodstuffs by high-performance liquid chromatography-photodiode array detection. Journal of Agricultural and Food Chemistry, v. 54, n. 3, p. 639-644, 2006.

DESIMONI, E.; BRUNETTI, B.; COSIO, M. S. Determination of Patent Blue V (E131) at a Nafion-modified glassy carbon electrode. Electroanalysis, v. 18, n. 3, p. 231-235, 2006.

DINC, E.; AKTAS, A. H.; USTUNDAG, O. New liquid chromatographicchemometric approach for the determination of sunset yellow and tartrazine in commercial preparation. Journal of AOAC International, v. 88, n. 6, p. 1748-1755, 2005.

ELHKIM, M. O. et al. New considerations regarding the risk assessment on Tartrazine. An update toxicological assessment, intolerance reactions and maximum theoretical daily intake in France. Regulatory Toxicology and Pharmacology, v. 47, n. 3, p. 308-316, 2007.

GENNARO, M. C.; ABRIGO, A.; CIPOLLA, G. High-performance liquid chromatography of food colours and its relevance in forensic chemistry. Journal of Chromatography A, v. 674, n. 1-2, p. 281-299, 1994.

GOSETTI, F. et al. Oxidative degradation of food dye E133 Brilliant Blue FCF Liquid chromatography-electrospray mass spectrometry identification of the degradation pathway. Jrournal of Chromatography A, v. 1054, n. 1-2, p. 379-387, 2004.
GÜLER, Z. Determination of synthetic colorants in confectionery and instant drink powders consumed in Turkey using UV/Vis spectrophotometry. Journal of Food Quality, v. 28, n. 1, p. 98-108, 2005.

KARAOGLAN, G. K. et al. Abilities of partial least-square (PLS-2) multivariate calibration in the analysis of quaternary mixture of food colors (E-110, E-122, E-124, E-131). Analytical Letters, v. 40, n. 10, p. 1893-1903, 2007.

KISELEVA, M. G.; PIMENOVA, V. V.; ELLER, K. I. Optimization of conditions for the HPLC determination of synthetic dyes in food. Journal of Analytical Chemistry, v. 58, n. 7, p. 685-690, 2003.

LAU, K. et al. Synergistic Interactions between Commonly Used Food Additives in a Developmental Neurotoxicity Test. Toxicological Sciences, v. 90, n. 1, p. 178-187, 2006.

LOPEZ-DE-ALBA, P. L.; LOPEZ-MARTINEZ, L.; DE-LEONRODRIGUEZ, L. M. Simultaneous determination of synthetic dyes tartrazine, allura red and sunset yellow by differential pulse polarography and partial least squares. A multivariate calibration method. Electroanalysis, v. 14, n. 3, p. 197-205, 2002.

MINIOTI, K. S.; SAKELLARIOU, C. F.; THOMAIDIS, N. S. Determination of 13 synthetic food colorants in water-soluble foods by reversed-phase high-performance liquid chromatography coupled with diode-array detector. Analytica Chimica Acta, v. 583, n. 1, p. 103-110, 2007.

PENMAN, K. G. et al. Bilberry Adulteration Using the Food Dye Amaranth. Journal of Agricultural and Food Chemistry, v. 54, n. 19, p. 7378-7382, 2006.

PERALTA-ZAMORA, P. et al. Spectrophotometric determination of organic dye mixtures by using multivariate calibration. Talanta, v. 47, n. 1, p.77-84, 1998.

PRABHAKARA RAO, P. G.; SATYANARAYANA, A.; RAO, D. G. Effect of storage on the stability of water soluble annatto dye formulation in a simulated Orange-RTS beverage model system. Lebensmittel-Wissenschaft und-Technologie, v. 35, n. 7 , p. 617-621, 2002.

PRADO, M. A. et al. Validation of methodology for simultaneous determination of synthetic dyes in alcoholic beverages by capillary electrophoresis. Journal of Chromatography A, v. 1136, n. 2, p. 231-236, 2006.

PRADO, M. A.; GODOY, H. T. Teores de corantes artificiais em alimentos determinados por cromatografia líquida de alta eficiência. Química Nova, v. 30, n. 2, p. 268-273, 2007.

RYVOLOVA, M. et al. Sensitive determination of erythrosine and other red food colorants using capillary electrophoresis with laser-induced fluorescence detection. Journal of Chromatography A, v. 1141, n. 2, p. 206-211, 2007.

SILVA, M. L. S. et al. Voltammetric determination of food colorants using a polyallylamine modified tubular electrode in a multicommutated flow system. Talanta, v. 72, n. 1, p. 282-288, 2007.

VIDOTTI, E. C. et al. Simultaneous determination of food dyes by first derivative spectrophotometry with sorption onto polyurethane foam. Analytical Sciences, v. 21, n. 2, p. 149-153, 2005.

VIDOTTI, E. C.; COSTA, W. F.; OLIVEIRA, C. C. Development of a green chromatographic method for determination of colorants in food samples. Talanta, v. 68, n. 3, p. 516-521, 2006.

VIDOTTI, E. C.; ROLLEMBERG, M. C. E. Espectrometria derivativa: uma estratégia simples para a determinação simultânea de corantes em alimentos. Química Nova, v. 29, n. 2, p. 230-233, 2006. 